\title{
Electronic structure, structural properties, and dielectric functions of IV-VI semiconductors: $\mathrm{PbSe}$ and PbTe
}

\author{
E. A. Albanesi \\ INTEC (CONICET-UNL), Guemes 3450, 3000 Santa Fe, Argentina \\ C. M. I. Okoye \\ Department of Physics and Astronomy, University of Nigeria, Nsukka, Nigeria \\ C. O. Rodriguez and E. L. Peltzer y Blanca \\ IFLYSIB, Grupo de Fisica del Solido, C.C. 565, La Plata 1900, Argentina \\ A. G. Petukhov \\ Physics Department, South Dakota School of Mines and Technology, Rapid City, South Dakota 57701-3995
}

(Received 12 January 2000)

\begin{abstract}
The electronic structure and frequency dependent dielectric function $\varepsilon(\omega)$ of rocksalt semiconductors $\mathrm{PbSe}$ and $\mathrm{PbTe}$ are investigated using the local density approximation (LDA) and the generalized gradient approximation as two different exchange and correlation approximations, within the full-potential linearized augmented plane-wave approach. Spin-orbit coupling has been incorporated in the study. The results are presented and compared with other recent calculations and experimental data. Structural properties are also obtained by means of calculations of total energy as a function of lattice parameters. The bulk structural parameters are sensitive to the choice of exchange and correlation approximation. The essential features of the band structure and density of states of $\mathrm{PbSe}$ and $\mathrm{PbTe}$ are reproduced by our calculations and agree quite well with available experimental results. The position of the minimum energy gap is correctly predicted, although the value of the gap is as usual, underestimated by the local density approximation with respect to the experimental data. This gap value is improved by the inclusion of the generalized gradient approximation. Also, we have calculated the real $\left[\varepsilon_{1}(\omega)\right]$ and imaginary $\left[\varepsilon_{2}(\omega)\right]$ parts of $\varepsilon(\omega)$ for both compounds, in the framework of the LDA scheme for exchange and correlation. The inclusion of spin-orbit coupling leads to a richer structure in both $\varepsilon_{1}(\omega)$ and $\varepsilon_{2}(\omega)$. The agreement with experimental results is satisfactory.
\end{abstract}

\section{INTRODUCTION}

The electronic structure, structural properties, and dielectric functions of the II-VI semiconductors have been previously investigated both experimentally and theoretically, by a variety of band structure techniques. ${ }^{1}$ The lead chalcogenides or lead salts PbSe and PbTe are IV-VI semiconductors. The study of these semiconductors is mainly motivated by their importance in long-wavelength sensor devices, diode lasers, and thermophotovoltaic energy converters. ${ }^{2}$ The lead salts crystallize in the rocksalt structure and are polar semiconductors. They possess some unusual electronic and structural properties, such as a very narrow energy gap, high carrier mobilities, high dielectric constants, and a positive temperature coefficient of the gap that may be unique among polar semiconductors. Furthermore, the band gaps have negative pressure coefficients, i.e., they decrease with increase in pressure, in contrast to what is observed in III-V and II-VI semiconductors. ${ }^{2,3}$ Also, PbSe and PbTe provide a case in which the minimum energy gap of a compound semiconductor increases as the atomic number of the anion increases. All these factors have contributed toward the interest in the fundamental study of this group of semiconductors.

There are presently in the literature several electronic structure studies of the lead salts $\mathrm{PbSe}$ and $\mathrm{PbTe}$. Some of them ${ }^{4-6}$ involve one or more adjustable parameters, obtain- ing different degrees of agreement with the experiments. Also, modern ab initio calculations have been performed, one involving a full potential linearized muffin-tin orbital using a Dirac relativistic version, ${ }^{7}$ obtaining good agreement with experimental bulk band structures, ${ }^{8}$ and another involving a general-potential linearized augmented plane-wave method, obtaining a good description of the electronic and structural properties. ${ }^{9}$

In this paper, we employ the full potential linearized augmented plane wave (FP-LAPW) method within the density functional theory as implemented in the WIEN97 code, ${ }^{10}$ which results in a very accurate scheme for electronic structure studies in solids. We perform calculations in order to study the electronic structure, structural properties, and dielectric functions of $\mathrm{PbSe}$ and $\mathrm{PbTe}$. It is known that, in many cases when the exchange and correlation effects are treated within the density functional approach using the standard local density approximation (LDA), it fails to yield satisfactory results and that using the generalized gradient approximation (GGA) gives better results. Also, the inclusion of spin-orbit interaction effects is known to improve the results of electronic structure studies when heavy elements are present, and for the lead salts, it seems to be particularly necessary. To this end, in this study we include the spin-orbit effect and employ two exchange and correlation approximations (LDA and GGA) in order to compare the results ob- 
TABLE I. Calculated equilibrium lattice constants $a_{e q}$, bulk modulus $B$, and pressure coefficient $B^{\prime}$.

\begin{tabular}{|c|c|c|c|c|c|c|c|c|}
\hline \multirow[b]{2}{*}{ Crystal } & \multicolumn{3}{|c|}{$a_{e q}(\mathrm{a} . \mathrm{u})$} & \multicolumn{3}{|c|}{$B$ (MBar) } & \multicolumn{2}{|c|}{$B^{\prime}$} \\
\hline & LDA & GGA & Expt & LDA & GGA & Expt & LDA & GGA \\
\hline $\mathrm{PbSe}$ (without spin-orbit coupling) & 11.410 & 11.745 & 11.5727 & 0.5954 & 0.4888 & & 4.7636 & 3.6449 \\
\hline $\mathrm{PbSe}$ (with spin-orbit coupling) & 11.419 & 11.777 & & 0.5726 & 0.4699 & $0.4-0.5^{\mathrm{a}}$ & 4.4156 & 4.6236 \\
\hline $\mathrm{PbTe}$ (without spin-orbit coupling) & 12.037 & 12.391 & 12.2114 & 0.5144 & 0.4030 & $0.4^{\mathrm{b}}$ & 5.5010 & 4.2746 \\
\hline $\mathrm{PbTe}$ (with spin-orbit coupling) & 12.057 & 12.420 & & 0.4982 & 0.3938 & & 5.7623 & 3.9163 \\
\hline
\end{tabular}

${ }^{\mathrm{a}}$ Reference 1 .

${ }^{\mathrm{b}}$ Reference 16.

tained using the two procedures.

This paper is organized as follows. In Sec. II, we give a brief description of the method used and details of the calculations. In Sec. III the total energy calculation as well as structural parameters, band structure, and density of states are presented. Also, results for the dielectric functions are presented and analyzed. Conclusions are drawn in the last section.

\section{DETAILS OF THE CALCULATIONS}

In this paper, we used the WIEN97 FP-LAPW code, the theoretical details of which are described by its authors elsewhere. ${ }^{10}$ The crystal structure of these IV-VI semiconductors is rocksalt with two atoms per unit cell. For the band structure and dielectric function calculations, we used the experimental values of the lattice constants, ${ }^{2,11}$ which are $6.124 \AA$ for $\mathrm{PbSe}$ and $6.462 \AA$ for PbTe. The implementation of the FP-LAPW method was carried out in the scope of the LDA using as the exchange-correlation potential the Perdew and Wang ${ }^{12}$ reparametrization of the Ceperley-Alder LDA data and the $\mathrm{GGA},{ }^{13}$ respectively. The Brillouin zone (BZ) integration was done with $72 k$ points in the irreducible part of the BZ. The energy as a function of lattice constant, the density of states, and the band structure were calculated with and without spin-orbit coupling using both LDA and GGA forms of exchange-correlation potential.

For the calculation of the bulk static properties of the compounds, we evaluated the total energy as a function of lattice constant. Around the region of the total energy minimum, the bulk modulus $B$, the pressure coefficient $B^{\prime}$, and the equilibrium lattice constant $a_{e q}$ were obtained by fitting the data to Murnaghan's equation of state. ${ }^{14}$ The total energy for each crystal was calculated using the two exchange and correlation schemes with and without spin-orbit coupling.

Once the band structure of a material is self-consistently obtained, one can obtain the frequency dependent dielectric function $\varepsilon(\omega)$ due to interband transitions. The imaginary part of the dielectric function $\operatorname{Im}[\varepsilon(\omega)]=\varepsilon_{2}(\omega)$ is treated
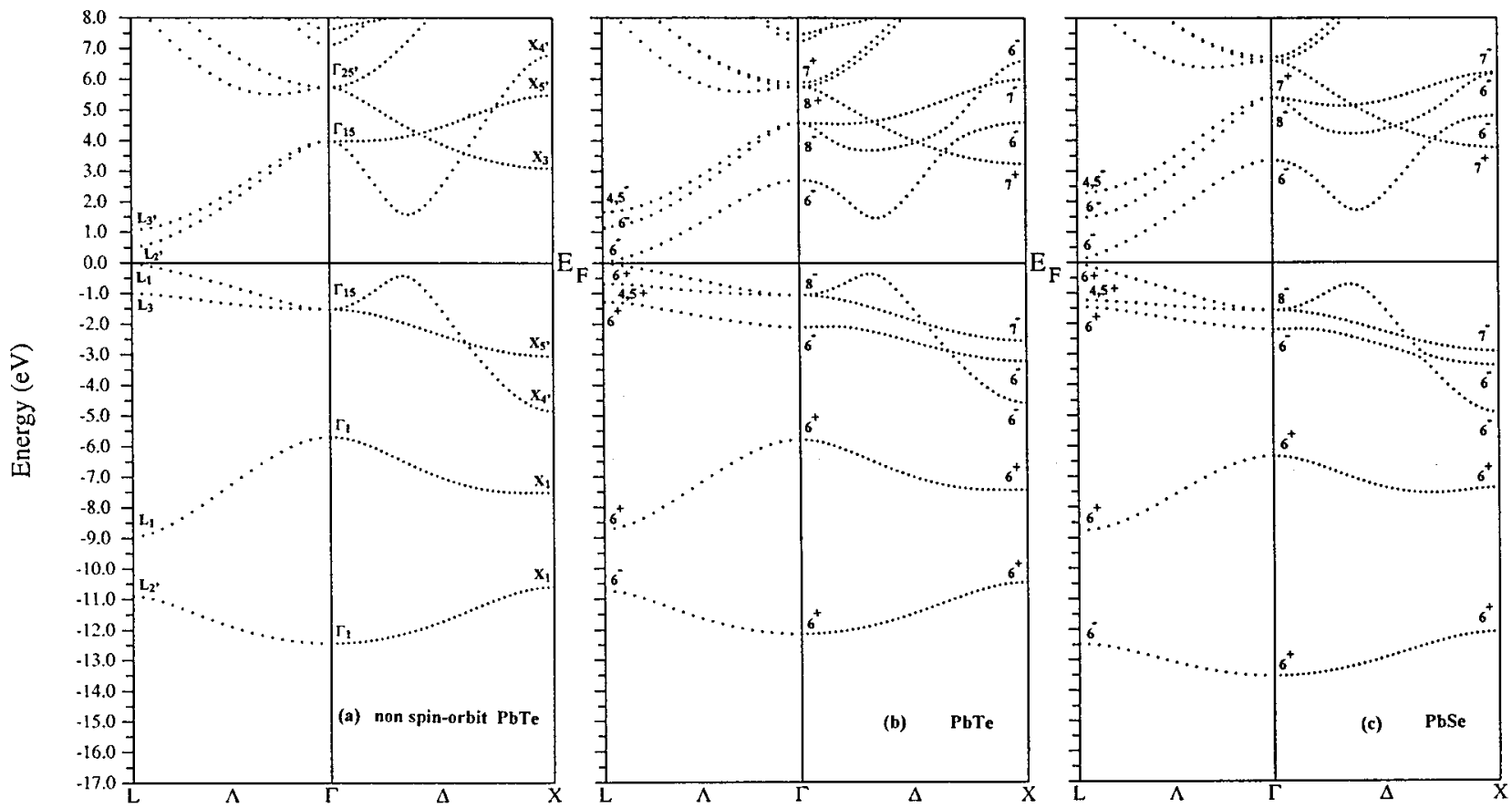

FIG. 1. Calculated electronic band structure for PbTe. (a) Scalar relativistic without spin-orbit interaction; (b) with spin-orbit interaction. In the latter case, the band spin-orbit splitting gives a better description of the band gap (aside from the underestimation of the LDA approximation). (c) Spin-orbit band structure for $\mathrm{PbSe}$. 
TABLE II. Calculated band gaps (in eV) at experimental lattice constants $a_{0}$. so indicate spin-orbit coupling.

\begin{tabular}{lccccc}
\hline \hline Crystal & LDA & GGA & LDA-so & GGA-so & Expt \\
\hline PbSe & 0.190 & 0.295 & 0.012 & 0.121 & $0.165^{\mathrm{a}}$ \\
PbTe & 0.570 & 0.730 & 0.028 & 0.160 & $0.190^{\mathrm{a}}$ \\
\hline
\end{tabular}

${ }^{\mathrm{a}}$ References 2 and 11.

by Ambroch-Draxl and $\mathrm{Abt}^{15}$ as

$$
\begin{aligned}
\operatorname{Im}[\varepsilon(\omega)]= & \varepsilon_{2}(\omega)=\left(4 \pi^{2} e^{2}\right) /\left(m^{2} \omega^{2}\right) \sum_{i, j} \int\langle i|M| f\rangle^{2} W_{i} \\
& \times\left(1-W_{f}\right) \delta\left(E_{f}-E_{i}-\omega\right) d^{3} k
\end{aligned}
$$

where $M$ is the dipole matrix, $i$ and $f$ are the initial and final states respectively, $W_{i}$ is the Fermi distribution function for the $i$ th state, and $E_{i}$ is the energy of the electron in the $i$ th state. From the imaginary part of the dielectric function we

TABLE III. Comparison between our results and available experimental values, for valence band critical point energies (in $\mathrm{eV}$ ),

\begin{tabular}{|c|c|c|c|}
\hline & Experimental $^{\mathrm{a}}$ & Experimental $^{\mathrm{b}}$ & LDA-so ${ }^{c}$ \\
\hline \multicolumn{4}{|c|}{$\mathrm{PbSe}$} \\
\hline$\Gamma_{8}^{-}$ & 1.45 & 1.8 & 1.53 \\
\hline$\Gamma_{6}^{-}$ & 2.2 & 2.5 & 2.17 \\
\hline$\Gamma_{6}^{+}$ & 6.25 & & 6.31 \\
\hline$\Gamma_{6}^{+}$ & 12.95 & & 13.51 \\
\hline$X_{7}^{-}$ & 2.75 & 3.2 & 2.88 \\
\hline$X_{6}^{-}$ & 3.3 & 3.7 & 3.34 \\
\hline$X_{6}^{-}$ & 4.45 & 5.1 & 4.89 \\
\hline$X_{6}^{+}$ & 7.7 & & 7.36 \\
\hline$X_{6}^{+}$ & 12.35 & & 12.04 \\
\hline \multicolumn{4}{|l|}{ Spin-orbit splitting } \\
\hline at $\Gamma$ & 0.75 & 0.6 & 0.63 \\
\hline \multicolumn{4}{|l|}{ Spin-orbit splitting } \\
\hline \multicolumn{4}{|c|}{$\mathrm{PbTe}$} \\
\hline$\Gamma_{8}^{-}$ & 1.0 & 1.3 & 1.06 \\
\hline$\Gamma_{6}^{-}$ & 2.1 & 2.5 & 2.12 \\
\hline$\Gamma_{6}^{+}$ & 6.35 & & 5.80 \\
\hline$\Gamma_{6}^{+}$ & 11.8 & & 12.16 \\
\hline$X_{7}^{-}$ & 2.45 & 2.5 & 2.55 \\
\hline$X_{6}^{-}$ & 3.55 & 3.4 & 3.23 \\
\hline$X_{6}^{-}$ & 4.5 & 4.5 & 4.60 \\
\hline$X_{6}^{+}$ & 7.85 & & 7.45 \\
\hline$X_{6}^{+}$ & 10.75 & & 10.50 \\
\hline Spin-orbit splitting & & & \\
\hline at $\Gamma$ & 1.10 & 1.15 & 1.06 \\
\hline $\begin{array}{l}\text { Spin-orbit splitting } \\
\text { at } X\end{array}$ & 1.10 & 0.9 & 0.70 \\
\hline
\end{tabular}
so indicates spin-orbit coupling.

${ }^{\mathrm{a}}$ Reference 8.

${ }^{\mathrm{b}}$ Reference 17.

${ }^{\mathrm{c}}$ This work.

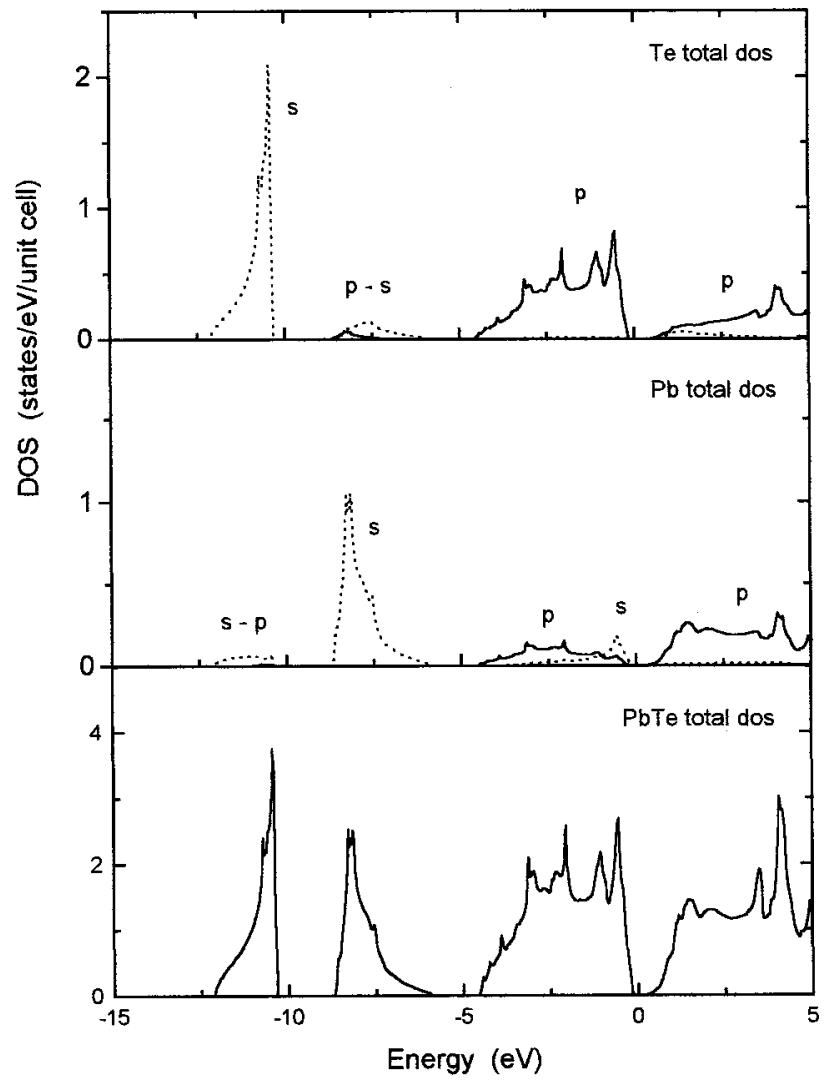

FIG. 2. Density of states for PbTe with spin-orbit interaction. Total (bottom plot), contribution from $\mathrm{Pb}$ atom (medium plot), and from the Te atom (top plot).

can extract the real part of the dielectric function $\operatorname{Re}[\epsilon(\omega)]$ $=\varepsilon_{1}(\omega)$ using the Kramers-Kronig relation in the form

$$
\operatorname{Re}[\varepsilon(\omega)]=\varepsilon_{1}(\omega)=1+\frac{2}{\pi} P \int_{0}^{k} \omega^{\prime} \varepsilon_{2}\left(\omega^{\prime}\right) d \omega^{\prime} /\left(\omega^{\prime 2}-\omega^{2}\right)
$$

where $P$ means the principal value of the integral. For the calculation of the optical properties, a dense mesh of $k$ points is required. The $\mathrm{BZ}$ integrations were done using the tetrahedron metod with $165 k$ points in the irreducible BZ without broadening.

\section{RESULTS AND DISCUSSION}

\section{A. Structural properties}

The ground state bulk properties of the crystals were obtained using the calculations of the total energy as a function of lattice constant. For each compound, the calculations were performed with and without spin-orbit coupling. The bulk properties from the total energy calculations are summarized in Table I together with available experimental data. The LDA underestimates the equilibrium lattice constant while the GGA, on the other hand, gives larger values. However, the deviation in the values predicted with either approximation scheme lies within $\pm 2 \%$ of the experimental values. One of the effects of the spin-orbit coupling is to lower the total energy by about 0.2 Ry in the compounds. For the bulk modulus $B$, the agreement with the experimental data is sat- 


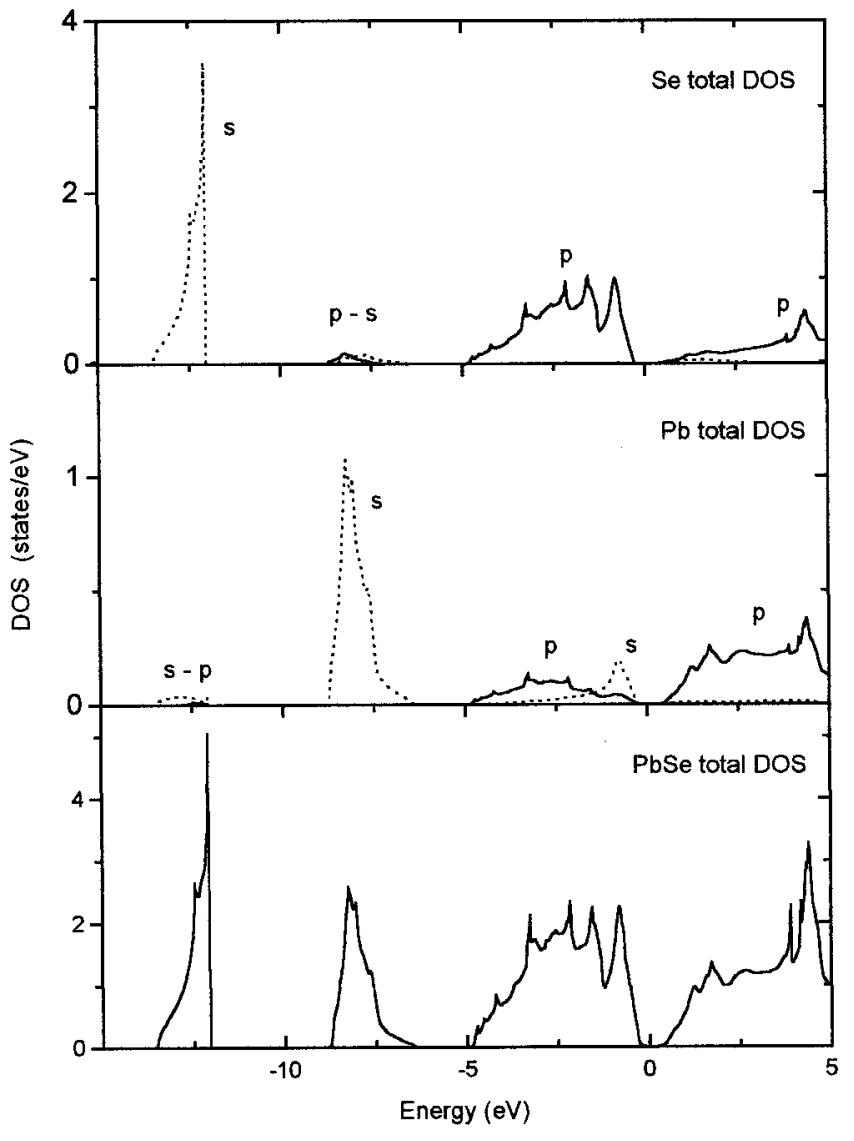

FIG. 3. Density of states for PbSe with spin-orbit interaction. Total (bottom plot), contribution from $\mathrm{Pb}$ atom (medium plot), and from the Se atom (top plot).

isfactory particularly for $\mathrm{PbSe}$, although there is a wide range of variation in the experimental data.

\section{B. Band structures and densities of states}

The calculated energy band structures along the principal symmetry directions for the two semiconductors PbSe and $\mathrm{PbTe}$ were calculated using the experimental values of the lattice constants for the respective crystals. In Fig. 1, we have displayed some of the band structures for comparison, performed in the LDA with and without the spin-orbit interaction. These band structures will be used in the discussion as representative of all the situations. Calculations were also done by treating the exchange and correlation effects using the GGA, and comparison of all band gaps is shown in Table II together with the experimentally determined values.

Even when no spin-orbit is considered, the scalar relativistic terms are included in our scheme. They are the massvelocity and the Darwin terms, which become important for heavy elements such as in our case the $\mathrm{Pb}$ cation. These contributions (which do not split the bands) are known to be rather dependent on the angular momentum character of the band, particularly for $p$-like states. As a consequence, the effects vary as a function of the $k$ vector, becoming more important for $k$ points with lower symmetry, which considerably affects the band gap value formed at the $L$ point. Our non-spin-orbit results show this behavior, giving a generally good account of the band structure, obtaining correctly a direct band gap at the $L$ point, although with an overesti- mated value of $0.570 \mathrm{eV}$. The maximum of the valence band (VBM) obtained at this point ( $L_{1}$ symmetry) results from hybridization with $18 \% \mathrm{~Pb} s$ - and $28 \% \mathrm{Te} p$-like character. The next lower band appears from the nearby $L_{3}$ symmetry valence band, having Te $p$-like character. The band forming the VBM gets closer to this one, merging together in going through the $\Lambda$ direction, resulting in degeneracy at the $\Gamma$ point. Deeper in energy appears another $L_{1}$ band with a strong $\mathrm{Pb} s$ character, which plays an important role in band gap formation, since this state interacts strongly with the VBM, both having the same symmetry $L_{1}$ at the edge of the Brillouin zone.

Our results also show, as expected, that these bands are modified and some of them split by the spin-orbit interaction, which will provide important modification at the $L$ point leading to an improved band gap value (in terms of the usual underestimation of the LDA formalism). In the double-group notation, the $L_{6}^{+}$state (derived from the former $L_{1}$ band) is the VBM, and close to it appear the time reversal doubly degenerate $L_{4}^{+}$and $L_{5}^{+}$states, and another $L_{6}^{+}$at lower energy (all three derived from the $L_{3}$ band). Deeper in energy is the $L_{6}^{+}$state corresponding to $s$-state $\mathrm{Pb}$. This band originates in the deep $L_{1}$ non-spin-orbit band, mantaining its shape and values with little change. In this double-group symmetry state labeling, it is easier to see the drastic reduction of the band gap value. The conduction band minimum (CBM) has $L_{6}^{-}$symmetry, while the next higher conduction band (CB) state is again $L_{6}^{-}$; the mutual repulsion moves down the CBM $L_{6}^{-}$state, shrinking the gap. The VBM is an $L_{6}^{+}$state and does not interact with these states; however, it is pushed up by the inner $L_{6}^{+} s$-state $\mathrm{Pb}$. As a result, the band gap decreases considerably with respect to the non-spin-orbit value. In Table II these results are compared at the experimental lattice constants for each compound.

The spin-orbit splitting of the bands at the $\Gamma$ point has simpler consequences than before, since there is no coupling between states, and the main effect is that both the $\Gamma_{15}$ valence band (VB) ( $p$-like Se and Te) and the $\Gamma_{15} \mathrm{CB}$ states, separated by the band gap energy, are split into the $\Gamma_{8}^{-}$quadruplet and the $\Gamma_{6}^{-}$doublet. This VB splitting is $0.63 \mathrm{eV}$ for $\mathrm{PbSe}$ and $1.06 \mathrm{eV}$ for $\mathrm{PbTe}$, in close agreement with experimental values (see Table III). Both $\Gamma_{8}^{-}$states maintain about the same energies as their predecessor non-spin-orbit $\Gamma_{15}$ states. At the $X$ point, VB spin-orbit splitting occurs between $X_{7}^{-}$and $X_{6}^{-}$, being $0.5 \mathrm{eV}$ for PbSe and $0.70 \mathrm{eV}$ for PbTe. Our values are in very satisfactory agreement with available experimental results, specially with those from angleresolved photoemission by Hinkel et al. ${ }^{8}$ as we show in Table III. Also, the essential features of the band structure of $\mathrm{PbSe}$ and $\mathrm{PbTe}$ are reproduced by our calculations and agree quite well with results obtained in previous calculations, ${ }^{8}$ especially those using similar full potential procedures. ${ }^{9}$

In Fig. 2 we show the calculated total density of states for $\mathrm{PbTe}$, which presents three well defined regions below the Fermi energy. These regions correspond, respectively, to the first bunch of bands close to $E_{F}$, and to the two next deepest bands in Fig. 1. The region close to $E_{F}$ forming the edge of the valence band has a sharp pike originating in the Te $p$ states and to a lesser extent in the $\mathrm{Pb} s$ states, this last contribution forming the VBM. The bulk of this region extends 


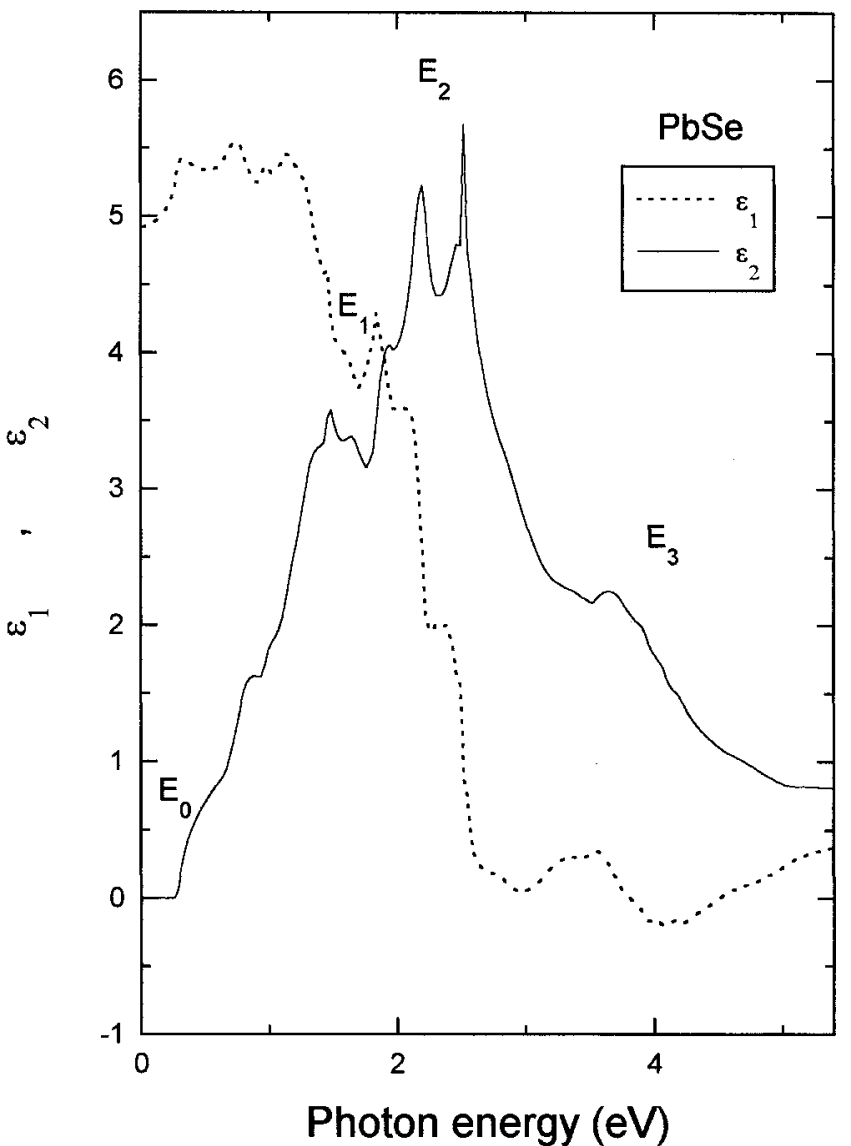

FIG. 4. Calculated real and imaginary parts of the dielectric function for $\mathrm{PbSe}$ with spin-orbit interaction. The values for the fine structure of the $E_{0}$ to $E_{3}$ regions are shown in Table IV and discussed in the text.

up to $-4.6 \mathrm{eV}$, and it is also formed mainly from the Te $p$ states with a small amount of $\mathrm{Pb} p$ character. The second region is narrower with a pronounced spike at $-8.29 \mathrm{eV}$ correspondig to the $\mathrm{Pb} s$ and some contribution of Te $s-p$ states. The last region is deepest in energy and slightly narrower than the previous one; it has a pronounced spike at $-10.4 \mathrm{eV}$ corresponding to the Te $s$ states. A small amount of $\mathrm{Pb} s$ also contributes to this peak. A rather similar situation is obtained for $\mathrm{PbSe}$ as shown in Fig. 3, although the first region close to $E_{F}$ is a little wider, and the deepest valence region corresponding to Se $s$ occurs at deeper energies, having its maximum peak at $-12.09 \mathrm{eV}$.

In order to show the effect of pressure on the energy gap in these semiconductors, we have also calculated the energy gaps at the equilibrium lattice constants obtained by using the two exchange and correlation schemes, namely, LDA and GGA. It is of interest to note that the expected negative pressure coefficient, which is experimentally observed, is obtained in our calculations. The trends in the values of the energy gaps at the corresponding lattice constants show this.

\section{Dielectric functions}

Different experimental techniques have been employed to measure optical properties in the lead chalcogenides, such as spectroscopic ellipsometry, ${ }^{3,18}$ which permits direct measurement of the real and imaginary parts of the pseudodielectric

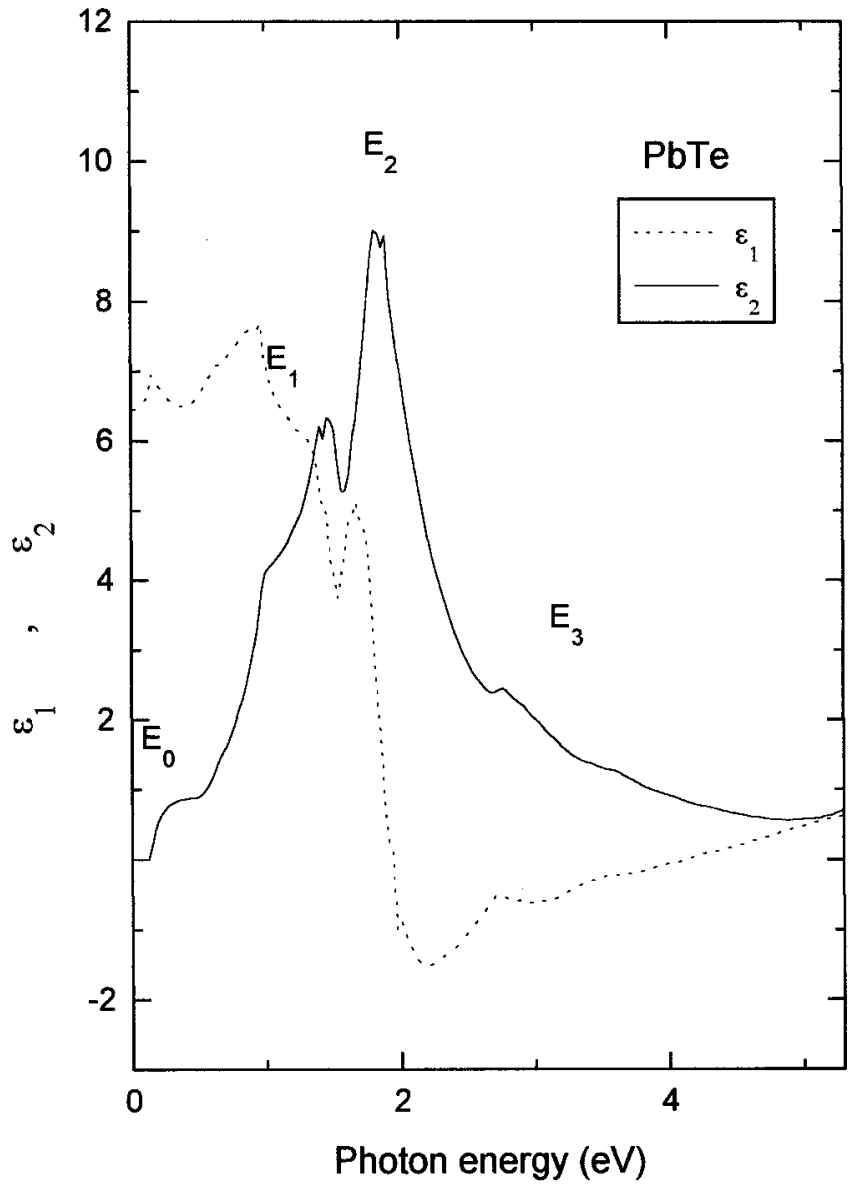

FIG. 5. Calculated real and imaginary parts of the dielectric function for $\mathrm{PbTe}$ with spin-orbit interaction. The values for the fine structure of the $E_{0}$ to $E_{3}$ regions are shown in Table $\mathrm{V}$ and discussed in the text.

function $\varepsilon(\omega)$; wavelength-modulation spectroscopy, ${ }^{19}$ electroabsorption and electroreflectance, ${ }^{20}$ and reflectance and transmission. ${ }^{21}$ The experimental results consist of rather broad peaks for these materials, and, as a consequence, different techniques give appreciable differences in the energy where the peaks are positioned. Following Cardona and Greenaway's $^{21}$ notation, the main characteristics of the $\varepsilon_{2}(\omega)$ spectra in the energy range of interest from $0 \mathrm{eV}$ to 5 $\mathrm{eV}$ are the threshold peak $E_{0}$, which should coincide with the band gap value, the first shoulder $E_{1}$, the highest peak in magnitude $E_{2}$, called the reflectivity peak which can show experimentally a fine structure to some extent, ${ }^{20}$ and the $E_{3}$ high-energy shoulder in the region of the main peak. The theoretical treatment show for both compounds that peaks possess structures, which are originated in the Van Hove singularities. ${ }^{11}$

We have calculated the real and imaginary parts of the frequency dependent dielectric function $\varepsilon(\omega)$ for $\mathrm{PbSe}$ and $\mathrm{PbTe}$ for photon energies up to $5 \mathrm{eV}$, using Eqs. (1) and (2), respectively, in the framework of the LDA scheme for exchange and correlation, with spin-orbit coupling. These real $\varepsilon_{1}(\omega)$ and imaginary $\varepsilon_{2}(\omega)$ parts are plotted in Fig. 4 for $\mathrm{PbSe}$ and in Fig. 5 for PbTe. The inclusion of spin-orbit coupling leads to a considerably greater number of peaks in both $\varepsilon_{1}(\omega)$ and $\varepsilon_{2}(\omega)$. 
TABLE IV. Calculated critical point energies for $\mathrm{PbSe}$, as discussed in the text, compared to collected available experimental results and other theoretical calculations. EPM means empirical pseudopotential method calculations.

\begin{tabular}{lccc}
\hline \hline & & \multicolumn{2}{c}{ Theory } \\
Region & Experiment $(\mathrm{eV})$ & $\operatorname{EPM}^{\mathrm{a}}(\mathrm{eV})$ & This work $(\mathrm{eV})$ \\
\hline$E_{0}$ & 0.31 & 0.17 & 0.26 \\
$E_{1}$ & $1.59^{\mathrm{c}}$ & 1.6 & 1.65 \\
& $1.97-2.18^{\mathrm{a}}$ & $1.9,2.3$ & 1.95 \\
& $1.6^{\mathrm{b}}$ & & \\
$E_{2}$ & $1.53-1.78^{\mathrm{c}}$ & & $2.19-2.51$ \\
& $2.65-3.12^{\mathrm{b}}$ & $2.7-2.9$ & \\
& $2.73^{\mathrm{b}}$ & & $3.63-4.20$ \\
$E_{3}$ & $3.1^{\mathrm{c}}$ & & \\
& $4.40-5.3^{\mathrm{a}}$ & $4.2-4.3$ & \\
& $4.10^{\mathrm{b}}$ & & \\
\hline
\end{tabular}

${ }^{a}$ Reference 19.

${ }^{b}$ Reference 3.

${ }^{\mathrm{c}}$ Reference 21 .

The results for both compounds are very similar, as could be expected from the similarities in the band structure from which the optical transitions take place. In Tables IV and V for $\mathrm{PbSe}$ and for $\mathrm{PbTe}$, respectively, we have collected available experimental results; it can be seen that our results agree quite well with them and also with other theoretical calculations. The main transitions originating from the peaks are assigned as follows: $L(5 \rightarrow 6)$ (in numbering the bands we have taken the usual notation, 1 as the deepest $s$ band) at $E_{0}$, $\Sigma(5 \rightarrow 6)[L(5 \rightarrow 7)]$ at $E_{1}, \Delta(5 \rightarrow 6)[\Sigma(5 \rightarrow 7)]$ at $E_{2}$, and $\Delta(4 \rightarrow 6)[\Sigma(4 \rightarrow 7)]$ at $E_{3}$.

The $E_{0}$ threshold appears at $0.29 \mathrm{eV}$ in PbSe and at 0.15 in PbTe. The next $E_{1}$ shoulder shows a fine structure of two peaks at 1.65 and $1.95 \mathrm{eV}$ in $\mathrm{PbSe}$ and at 1.02 and $1.46 \mathrm{eV}$ in PbTe. After this, the main $E_{2}$ peak in $\varepsilon_{2}(\omega)$ appears. This is the most important feature of the spectrum, since it can give a measure of the absolute value of the reflectivity for a given sample. Also, in the framework of spectroscopic ellipsometry, ${ }^{3,18}$ it permits one to recognize the cleanest surface, which provides a more bulklike dielectric function. Fine structure can be found also for the third peak $E_{3}$, at 3.63 and $4.20 \mathrm{eV}$ for $\mathrm{PbSe}$ and 2.76 and $3.55 \mathrm{eV}$ for PbTe. After going through this peak, the calculated $\varepsilon_{2}(\omega)$ decreases with increasing energy. The peaks in the spectra are better defined in $\mathrm{PbTe}$, characterized by the fact that both maximum fine structure critical points at $E_{2}$ occur at almost the same energy (only $0.09 \mathrm{eV}$ apart), resulting in a sharper and narrower $\varepsilon_{2}(\omega)$ spectrum as can be seen by comparing Figs. 4 and 5 . Also, the peaks corresponding to $E_{1}$ are more separated in energy from the $E_{2}$ peaks in $\mathrm{PbTe}$ than in $\mathrm{PbSe}$, improving definition. In contrast, the $\mathrm{PbSe} \varepsilon_{2}(\omega)$ spectra is broader and have more relief.

For the real dielectric function, the most important quantity is the zero frequency limit $\varepsilon_{1}(0)$ which is the electronic part of the static dielectric constant. ${ }^{22}$ From the $\varepsilon_{1}(\omega)$ curves, approximate values of the electronic part of the static dielectric constant in the zero frequency limit can be ob-
TABLE V. Calculated critical point energies for $\mathrm{PbTe}$, as discussed in the text, compared to collected available experimental results and other theoretical calculations. EPM means empirical pseudopotential method calculations.

\begin{tabular}{lccc}
\hline \hline & & \multicolumn{2}{c}{ Theory } \\
Region & Experiment $(\mathrm{eV})$ & $\operatorname{EPM}^{\mathrm{a}}(\mathrm{eV})$ & This work (eV) \\
\hline$E_{0}$ & & 0.19 & 0.15 \\
$E_{1}$ & $1.08^{\mathrm{b}}$ & $1.07-1.27$ & $1.02-1.45$ \\
& $1.25-1.60^{\mathrm{d}}$ & & \\
$E_{2}$ & $1.98-2.2^{\mathrm{c}}$ & $2.02-2.03$ & $1.81-1.89$ \\
& $2.1-2.3^{\mathrm{a}}$ & & \\
& $2.02^{\mathrm{b}}$ & & \\
$E_{3}$ & $3.3^{\mathrm{a}}$ & $2.78-3.1$ & \\
& $3.5^{\mathrm{c}, \mathrm{d}}$ & & \\
& $3.25^{\mathrm{b}}$ & & \\
& & & \\
\hline \hline
\end{tabular}

${ }^{\mathrm{a}}$ Reference 19 .

${ }^{\mathrm{b}}$ Reference 18 .

${ }^{\mathrm{c}}$ Reference 21.

${ }^{\mathrm{d}}$ Reference 20 .

tained, provided that the absolute magnitudes have been adjusted from experiments. Our $\varepsilon_{1}(\omega)$ curves agree with those obtained by Kohn et al. ${ }^{19}$

\section{CONCLUSIONS}

We have presented a systematic study of the electronic and structural properties, as well as the dielectric functions, of the lead salts PbSe and PbTe, using the all-electron FPLAPW method with different forms of exchange and correlation potentials. We have studied the structural properties, comparing the results obtained with the LDA and the GGA, without and including spin-orbit interaction. In any case, the inclusion of spin-orbit coupling in the calculations significantly improved the agreement of the band structure and dielectric functions with experiments. Both schemes, LDA and GGA, give good agreement with experimental values, GGA being better for the structural properties. We compared the band-gaps in the same manner, obtaining the usual result that the LDA underestimates the energy gap, while on the other hand, the GGA improves these results making the GGA spin-orbit band gaps comparable with their experimental values.

The energy band structures and density of states are very well described by our calculations performed in the framework of the LDA, as we have shown in Sec. III B, applying them to the calculation of dielectric functions for both materials. We have explained the main features of available experimental data, as well as compared our results with other theoretical calculations.

\section{ACKNOWLEDGMENTS}

The authors acknowledge finnancial support from the Consejo Nacional de Investigaciones Científicas y Técnicas de Argentina (CONICET). One of the authors (C.M.I.O.) is grateful to the TWAS and the CONICET for providing financial support under the TWAS South-South Fellowship program during his stay at IFLYSIB, La Plata, Argentina. 
${ }^{1}$ G. Nimtz, B. Schlicht, and B. Dornhaus, Narrow Gap Semiconductors: Springer Tracts in Modern Physics (Springer, New York, 1983), and references therein.

${ }^{2}$ R. Dalven, in Solid State Physics, edited by H. Ehrenreich, F. Seitz, and D. Turnbull (Academic Press, New York, 1973), Vol. 28, p. 179.

${ }^{3}$ N. Suzuki, K. Sawai, and S. Adachi, J. Appl. Phys. 77, 1249 (1995).

${ }^{4}$ G. Martinez, M. Schlüter, and M. Cohen, Phys. Rev. B 11, 651 (1975).

${ }^{5}$ K. Rabe and J. Joannopoulos, Phys. Rev. B 32, 2302 (1985).

${ }^{6}$ J.A. Valdivia and G.E. Barberis, J. Phys. Chem. Solids 56, 1141 (1995).

${ }^{7}$ N.E. Christensen, Int. J. Quantum Chem. 25, 233 (1984); see also C. Godreche, J. Magn. Magn. Mater. 29, 262 (1982), and references therein.

${ }^{8}$ V. Hinkel, H. Hoak, C. Mariana, L. Sorba, K. Horn, and N.E. Christensen, Phys. Rev. B 40, 5549 (1989).

${ }^{9}$ Su-Hai Wei and A. Zunger, Phys. Rev. B 55, 13605 (1997).

${ }^{10}$ P. Blaha, K. Schwarz, and J. Luitz, computer code WIEN97 (Vienna University of Technology, 1997). Improved and updated version published by P. Blaha, K. Schwarz, P. Sorantin, and S.B. Rickey, Comput. Phys. Commun. 59, 399 (1990).

${ }^{11}$ M. L. Cohen and J. R. Chelikowsky, Electronic Sructure and Optical Properties of Semiconductors, 2nd ed., Vol. 75 of Springer Series in Solid State Sciences, edited by M. Cardona (Springer-Verlag, Berlin, 1989).

12 J.P. Perdew and Y. Wang Phys. Rev. B 45, 13244 (1992).

${ }^{13}$ J.P. Perdew, S. Burke, and M. Ernzerhof, Phys. Rev. Lett. 77, 3865 (1996).

${ }^{14}$ F.D. Murnaghan, Proc. natl. Acad. Sci. USA 30, 244 (1944).

${ }^{15} \mathrm{C}$. Ambroch-Draxl and R. Abt (unpublished).

${ }^{16}$ A.J. Miller, G.A. Saunders, and Y.K. Yogurtcu, J. Phys. C 14, 1569 (1981).

${ }^{17}$ Th. Grandke, L. Levy, and M. Cardona, Phys. Rev. B 18, 3847 (1978).

${ }^{18}$ N. Suzuki, and S. Adachi, J. Appl. Phys. 33, 193 (1994).

${ }^{19}$ S.E. Kohn, P.Y. Yu, Y. Petroff, Y.R. Shen, Y. Tsang, and M.L. Cohen, Phys. Rev. B 8, 1477 (1973).

${ }^{20}$ D.E. Aspnes and M. Cardona, Phys. Rev. 173, 714 (1968).

${ }^{21}$ M. Cardona and D.L. Greenaway, Phys. Rev. 133, A1685 (1964).

${ }^{22}$ Yong-Niam Xu and W.Y. Ching, Phys. Rev. B 48, 4335 (1993). 